Portland State University

PDXScholar

$1-15-1982$

\title{
Dielectric Constant in Fluids of Classical Deformable Molecules
}

John D. Ramshaw

Portland State University, jdramshaw@yahoo.com

Follow this and additional works at: https://pdxscholar.library.pdx.edu/phy_fac

Part of the Physics Commons

Let us know how access to this document benefits you.

\section{Citation Details}

J.D. Ramshaw, "Dielectric constant in fluids of classical deformable molecules," J. Chem. Phys. 76, 1183 (1982)

This Article is brought to you for free and open access. It has been accepted for inclusion in Physics Faculty Publications and Presentations by an authorized administrator of PDXScholar. Please contact us if we can make this document more accessible: pdxscholar@pdx.edu. 
4(a) J. C. Rasaiah and H. L. Friedman, J. Chem. Phys. 48, 2742 (1968); (b) J. C. Rasaiah and H. L. Friedman, J. Chem. Phys. 50, 3965 (1969).

${ }^{5}$ The meaning of symbols except for the step function $\theta(x)$ is the same as in Ref. 4.

${ }^{6} \mathrm{~V}$. I. Krylov, Approximate Calculations of Integrals (Macmillan, New York, 1962).

${ }^{7}$ Extensive tabulations of these results will be made available to interested people.

${ }^{8}$ D. N. Card and J. P. Valleau, J. Chem. Phys. 52, 6232
(1970).

's. Ciccariello, D. Gazzillo, and C. Dejak, Mol. Phys. 39, 597 (1980).

${ }^{10} \mathrm{~J}$. C. Rasaiah, J. Chem. Phys. 56, 3071 (1972).

${ }^{11} \mathrm{P}$. J. Rossky, J. B. Dudowicz, B. L. Tembe, and H. L. Friedman, J. Chem. Phys. 73, 3372 (1980).

${ }^{12}$ This is strictly true only with "standard" initializations of the HNC iterative solution (e.g. Ref. 4). More general initializations are discussed in: P. J. Rossky and H. L. Friedman, J. Chem. Phys. 72, 5694 (1980).

\title{
Dielectric constant in fluids of classical deformable molecules $^{\text {a) }}$
}

\author{
John D. Ramshaw \\ Theoretical Division, University of California, Los Alamos National Laboratory, Los Alamos, New \\ Mexico 87545 \\ (Received 25 August 1981; accepted 6 October 1981)
}

Recently there has been a growing interest in purely classical descriptions of dielectric fluids composed of deformable (polarizable) molecules. ${ }^{1-5}$ In such treatments, classical statistical mechanics is applied not only to translational and rotational coordinates, but also to the internal molecular coordinates associated with polarizability. The main drawback of such an approach is that it implies incorrect dispersion forces between the molecules. 3, 4,6 However, one may compensate for this error by an appropriate choice of the intermolecular potential. ${ }^{3}$

Much of the work cited has been concerned with simple approximations (such as the mean spherical and related approximations) in which analytically tractable results may be obtained. It may be of some complementary interest to point out that the restriction to a purely classical description enables one to obtain very general expressions for the dielectric constant $\epsilon$ which are remarkably simple in form. Two such expressions are given below. These expressions are valid for an arbitrary molecular model (provided only that internal molecular degrees of freedom are treated classically), and they apply to fluid mixtures as well as pure fluids.

The first expression relates $\epsilon$ to dipole moment correlations. It follows easily from the general formula ${ }^{7}$ $(\epsilon-1)(2 \epsilon+1) /(4 \pi \epsilon)=\int d \mathbf{r} \mathbf{U}: \mathbf{K}(\mathbf{r})$, where $\mathbf{K}(\mathbf{r})$ is the $d y-$ adic kernel that relates the polarization $P(r)$ to the external electric field $E_{0}(r)$ in an infinite system, and $U$ is the unit dyadic. In a purely classical system, one readily finds that $K(\mathbf{r})=\beta\langle\mathbf{P}(\mathbf{r}) \mathbf{P}(0)\rangle$, where the angular brackets denote an equilibrium ensemble average in the unperturbed system, and $\beta=1 / k T$. We thereby obtain

$$
\frac{(\epsilon-1)(2 \epsilon+1)}{4 \pi \epsilon}=\beta \int d r\langle P(0) \cdot P(r)\rangle \text {. }
$$

The second expression relates $\epsilon$ to charge density correlations. It follows from the purely classical for- mula $(\epsilon-1) /(4 \pi \epsilon)=\beta Q$, where $Q$ is the limit as $\mathbf{k}-0$ of the quantity

$$
|\mathbf{k}|^{-2} \int d \mathbf{r} \exp (i \mathbf{k} \cdot \mathbf{r})\langle\rho(0) \rho(\mathbf{r})\rangle
$$

and $\rho(r)$ is the microscopic charge density at the point $r$. (This formula is easily verified by an argument similar to that which is used to obtain $\epsilon(k)$ in ionic fluids. ${ }^{8}$ ) The limit may be taken using L'Hôpital's rule, with the result

$$
\frac{\epsilon-1}{4 \pi \epsilon}=-\frac{1}{6} \beta \int d \mathbf{r}|\mathbf{r}|^{2}\langle\rho(0) \rho(\mathbf{r})\rangle .
$$

In order for $\epsilon$ to be finite, the correlation functions $\langle\mathbf{P}(0) \cdot \mathbf{P}(\mathbf{r})\rangle$ and $\langle\rho(0) \rho(\mathbf{r})\rangle$ must be short ranged. It seems likely ${ }^{1,9}$ that both of these functions will in general decay like $|\mathbf{r}|^{-6}$ as $|\boldsymbol{r}| \rightarrow \infty$.

The status of Eqs. (1) and (2) is somewhat difficult to characterize. Equation (1) is by far the more familiar, and expressions equivalent to it are encountered rather frequently (e.g., see Felderhof ${ }^{10}$ ). Equation (2) is much less well known, in spite of the fact that in essence it is simply a limiting case of a relation which is well known in another context. But while they are not really new results, the equations themselves and the full extent of their generality do not seem to be widely appreciated, especially in the case of Eq. (2). It therefore seems worthwhile to focus greater attention on these relations, and, in particular, to emphasize their applicability in the context of present concern. ${ }^{1-5}$

The great generality of Eqs. (1) and (2) makes them useful as starting points for derivations of more specialized expressions. In the case of $\mathrm{Eq}$. (1), the first step is to write

$$
\mathbf{P}(\mathbf{r})=\sum_{k} \mu_{k} \delta\left(\mathbf{r}-\mathbf{r}_{k}\right),
$$


where $r_{k}$ and $\mu_{k}$ are the position and dipole moment, respectively, of molecule $k$. Substitution into Eq. (1) then yields, for a pure fluid,

$$
\begin{aligned}
\frac{(\epsilon-1)(2 \epsilon+1)}{4 \pi \epsilon} & =\beta n \int d \mu|\mu|^{2} s(\mu) \\
& +\beta n^{2} \int d \mathbf{r} d \mu d \mu^{\prime} \mu \cdot \mu^{\prime} h\left(\mathbf{r}, \mu, \mu^{\prime}\right),
\end{aligned}
$$

where $s(\mu)$ and $h\left(\mathbf{r}, \mu, \mu^{\prime}\right)$ are the singlet and pair dipole moment correlation functions introduced by Pratt, ${ }^{2}$ and $n$ is the molecular number density. [Note that a factor of two should be inserted on the right side of Pratt's Eq. (2.6). I Equation (3) may be regarded as the generalization of the Kirkwood equation ${ }^{11}$ to classical deformable molecules.

Similarly, for Eq. (2) one writes $\rho(\mathbf{r})=\sum_{k} \rho_{k}(\mathbf{r})$, where $\rho_{k}(\mathbf{r})$ is the charge density of molecule $k$ at the point $\mathbf{r}$. Singlet and pair charge density correlation functions then appear. To proceed further, the form of $\rho_{k}(\mathbf{r})$ must be specified. The simplest choice of $\rho_{k}(\mathbf{r})$ is that appropriate to a collection of point charges; i.e., $\rho_{k}(\mathbf{r})$ $=\sum_{\alpha} z_{\alpha k} \delta\left(\boldsymbol{r}-\mathbf{r}_{k \alpha}\right)$, where $\mathbf{r}_{k \alpha}$ is the position of point charge $\alpha$ in molecule $k, z_{\alpha k}$ is its charge, and $\sum_{\alpha} z_{\alpha k}=0$. This choice, of course, leads naturally to a site-site correlation function description, and provides perhaps the simplest route to the site-site expressions for $\epsilon .{ }^{1,9}$

a) Work performed under the auspices of the United States Department of Energy.

${ }^{1}$ D. Chandler, J. Chem. Phys. 67, 1113 (1977).

${ }^{2}$ L. R. Pratt, Mol. Phys. 40, 347 (1980).

${ }^{3}$ J. S. Høye and G. Stell, J. Chem. Phys. 72, 1597 (1980).

${ }^{4}$ J. S. Hoye and G. Stell, J. Chem. Phys. 73, $461(1980)$.

${ }^{5}$ J. S. Høye and G. Stell, J. Chem. Phys. 75, 3565 (1981).

${ }^{6}$ W. F. Brown, Jr., in Handbuch der Physik, edited by S. Flügge (Springer, Berlin, 1956), Vol. 17, p. 1.

${ }^{7}$ M. S. Wertheim, Mol. Phys. 26, 1425 (1973).

${ }^{8}$ N. H. March and M. P. Tosi, Atomic Dynamics in Liquids (Wiley, New York, 1976).

${ }^{9}$ J. S. Høye and G. Stell, J. Chem. Phys, 65, 18 (1976),

${ }^{10}$ B. U. Felderhof, J. Chem. Phys, 67, 493 (1977).

${ }^{11}$ J. G. Kirkwood, J. Chem. Phys. 7, 911 (1939).

\title{
Single vibronic emissions from NO $B^{2} \Pi\left(v^{\prime}=7\right)$ and $\mathrm{O}_{2} B^{3} \Sigma_{u}^{-}$ $\left(V^{\prime}=4\right)$ excited by $193 \mathrm{~nm}$ ArF laser
}

\author{
Kazuhiko Shibuyaa) and F.Stuhl \\ Ruhr Universität, Physikalische Chemie I, D-4630 Bochum, West Germany \\ (Received 19 May 1981; accepted 8 September 1981)
}

Previously, powerful excimer lasers have been successfully used to study unfavorable absorption processes such as multiple photon dissociation. Often the products generated in these processes are monitored by efficient detection methods such as fluorescence and laser induced fluorescence of the dissociation fragments. In this note, we would like to report on weak single photon absorption processes which selectively populate single vibronic levels in diatomic molecules. Since this excitation was found to lead to predissociative states of $\mathrm{NO}$ and $\mathrm{O}_{2}$, the subsequent fluorescence is inefficient.

The photon energy of the ArF laser corresponds to the absorption of the NO $B^{2} \Pi\left(v^{\prime}=7\right)-X^{2} \Pi\left(v^{\prime \prime}=0\right)$ and $A^{2} \Sigma$ $\left(v^{\prime}=3\right)-X^{2} \Pi\left(v^{\prime \prime}=0\right)$ transitions. The $F_{1}$ component of the $B\left(v^{\prime}=7\right)-X\left(v^{\prime \prime}=0\right)$ absorption of NO originates at $52347 \mathrm{~cm}^{-1}$ and the spectrum is shaded towards the red. From available spectroscopic data, ${ }^{1,2}$ NO $X\left(v^{\prime \prime}=0\right)$ is expected to be excited to the $B\left(v^{\prime}=7\right)$ state via the absorption lines $R_{11}(30.5$ to 32.5$), P_{11}(27.5$ to 29.5$)$, $Q_{11}(28.5$ to 31.5$), R_{22}(28.5$ to 30.5$), P_{22}(25.5$ to 27.5$)$, and $Q_{22}(26.5$ to 29.5$)$. On the other hand, the $A^{2} \Sigma\left(v^{\prime}=3\right)$ $-X^{2} \Pi\left(v^{\prime \prime}=0\right)$ absorption is shaded towards the blue with an origin of $F_{1}$ at $51130 \mathrm{~cm}^{-1}$. Energy matching of this absorption requires the preparation of NO $A\left(v^{\prime}=3\right)$ from higher rotational levels of the NO $X\left(v^{\prime \prime}=0, J^{\prime \prime} \sim 50.5\right)$ state. The population of rotationally excited NO with $J^{\prime \prime} \sim 50.5$, however, is about $10^{6}$ times smaller than that of $J^{\prime \prime}=28.5$ at $(298 \pm 2) \mathrm{K}$. Therefore, preferably the formation of highly excited rotational levels $\left(J^{\prime}=26.5\right.$ to 33.5 for $F_{1}$ and 24.5 to 31.5 for $\left.F_{2}\right)$ of the $B\left(v^{\prime}=7\right)$ state is to be expected in the irradiation of NO by an ArF laser.

According to the well-known absorption of the Schumann-Runge bands $\mathrm{O}_{2}$ molecules are excited by light from an ArF laser to the $B^{3} \Sigma_{u}^{-}\left(v^{\prime}=4\right)$ state via several rotational lines of the $P\left(N^{\prime \prime}=15,17\right.$, and 19) and $R\left(N^{\prime \prime}\right.$ $=17,19$, and 21$)$ branches $^{3}$

In the present experiments, an unfocused ArF laser (Lambda Physik, EMG 501) was operated at $3 \mathrm{~Hz}$ producing 100 to $150 \mathrm{~mJ}$ per pulse at $193.34 \mathrm{~nm}$ (FWHM $0.5 \mathrm{~nm}$ ). The fluorescence generated upon irradiation of NO (purity $>99.85 \%$ ) or $\mathrm{O}_{2}$ (purity $>99.5 \%$ ) was dispersed by a monochromator ( $0.5 \mathrm{~m}$ Minuteman) using a grating blazed at $300 \mathrm{~nm}$. The intensity of the fluorescence was measured by a photomultiplier (EMI 9789 $\mathrm{QB})$, and the signal was averaged by a boxcar integrator (PAR 162/165).

The observed NO fluorescence is shown in Fig. 1 together with the distribution of the fluorescence intensity estimated from theoretical values of Franck-Condon factors for the $B^{2} \Pi\left(v^{\prime}=7\right) \rightarrow X^{2} \Pi\left(v^{\prime \prime}\right)$ progression. ${ }^{4}$ The agreement between experiment and calculation appears to be reasonably good considering previous results ob- 